\title{
Three-year anniversary of Science China Materials- Thank you to our authors, reviewers, and readers!
}

On behalf of Science China Materials, we extend our gratitude to all authors, reviewers, and readers for your continuing support to the journal. In the coming new year, we will strive to provide a broader service for the scientists working in the area of materials science and engineering.

The year 2017 has been a milestone to Science China Materials, which has been included by the SCI and Ei Compendex and has also acquired its first impact factor of 3.956. The highlights of the activities that we had carried out during the last year are listed below, which we anticipate to be interesting and useful to you.

We received more than 450 submissions and published more than 100 original articles and review papers in 2017. We also kept track of the papers published in Science China Materials that had been cited almost 1,000 times.

The following two Special Issues have been organized and published:

- Biomaterial Foundations of Therapeutic Delivery, prepared by Guest Editors Prof. Hasan Uludağ and Prof. Gu Ning, presents a collection of manuscripts describing the most recent advances in biomaterials for drug delivery systems.

- Tsinghua-NTU Bilateral Workshop for Young Scientists, prepared by Guest Editor Prof. Xun Wang, presents some recent research progress made in nanoscience and optoelectronic materials, which has been achieved by researchers from Tsinghua University and Nanyang Technological University.

In addition, we have invited experts in a few highly interesting and significant areas to review their research, including the design, preparation, and toxicity of nanostructured materials; energy conversion nanomaterials, carbon-based supercapacitors, and aluminum-based battery systems; heterogeneous catalysts; and degradable biomaterials. These reviews, looking back at the progress and prospecting their future, are intended to cover recent advances in materials science and technology and could expectedly guide the future research.

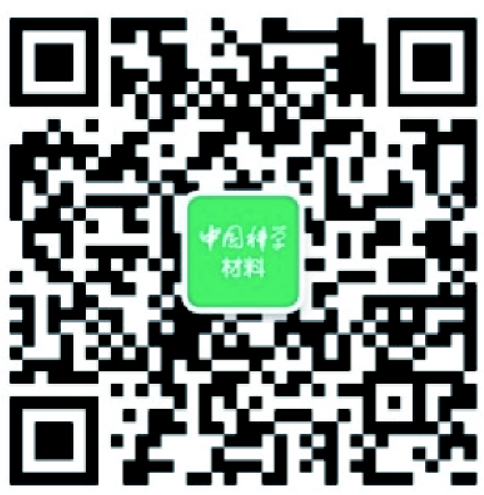

A WeChat public account has been applied and is running actively, where the latest published papers are promoted and introduced in a concise manner. Quick Response Code is attached here, and we welcome you to join us.

You can also keep in touch with us on Facebook and in person at conferences for discussing the new information about our journal, including the latest research papers, editorial policies, and interesting issues in materials science and technology.

From 2018 onward, we have 18 new colleagues joining the editorial board and the advisory board, who are leading scientists and rising research stars in the field of materials science. They would also provide constructive advice to our authors and readers and extend the frontier and scope of our original articles. Their names have been announced on the website of the journal. We welcome them to Science China Materials.

On the eve of 2018, we express our best wishes to you through this platform and look forward to your constructive advice and continuing support.

Editor-in-Chief

Prof. Yadong Li

Department of Chemistry, Tsinghua University Email: ydli@mail.tsinghua.edu.cn 